Rev. Saúde públ., S. Paulo, 24(4): 332-6, 1990

\title{
TRATAMENTO DA HIPERTENSÃO E DECLÍNIO DA MORTALIDADE POR ACIDENTES VASCULARES CEREBRAIS
}

\author{
José Eluf Neto* \\ Paulo Andrade Lotufo** \\ Cecilia Amaro de Lólio***
}

\begin{abstract}
ELUF NETO, J. et al. Tratamento da hipertensão e declínio da mortalidade por acidentes vasculares cerebrais. Rev. Saúde públ., S. Paulo, 24: 332-6, 1990.

RESUMO: É discutida a possível relação causa-efeito entre o declínio da mortalidade por acidentes vasculares cerebrais (AVC) e o melhor tratamento da hipertensão arterial. Para isto são revistos criticamente na literatura internacional os possíveis artefatos estatísticos para a enumeração dos AVCs, a letalidade e a incidência de AVC, a prevalência de outros fatores de risco como hipercolesterolemia, tabagismo, ingestão de sódio, potássio e álcool, a obesidade e também a contribuição da assistência médica.
\end{abstract}

DESCRITORES: Distúrbios cerebrovasculares, mortalidade. Previsð̃es. Hipertensão, prevenção.

Nas duas últimas décadas tem-se descrito declínio da mortalidade por acidentes vasculares cerebrais (AVC), em muitos países. O declínio tem ocorrido em nações industrializadas ${ }^{33}$ e em países em desenvolvimento ${ }^{24}$, quer tenham altas taxas de mortalidade (Japão), ou baixas taxas (Estados Unidos) ${ }^{33}$. A mortalidade decresceu em várias regiōes dos países analisados 7 , em ambos os sexos ${ }^{33}$, em diferentes grupos étnicos e em todos os grupos de idade $^{36}$. Entretanto, em alguns países, a mortalidade por AVC tem aumentado em anos recentes: a Polônia, a Hungria e a Bulgária têm mostrado taxas crescentes em ambos os sexos; e a Romênia e a Iugoslávia têm apresentado elevação das taxas de mortalidade para homens ${ }^{33}$.

No Brasil ${ }^{29}$, a mortalidade por AVC é bastante alta, sendo que em 1985 ocorreram 73.205 6́bitos por estas afecçб̃es $(9,3 \%$ do total de 6 bitos). Para o mesmo ano, 7,9\% dos 6́bitos foram devidos à doença isquêmica do coração (doença coronária). Já para o Estado de São Paula no mesmo ano, a proporção de obitos por doença coronária era ligeiramente superior $(11,2 \%)$ a de óbitos por AVC $(10,6 \%)$. Comparando a mortalidade por AVC no Município de São Paulo com a observada em 27 países industrializados, para o ano de 1980, embora ela venha declinando, as taxas ajustadas por idade observadas para São Paulo se posicionaram em terceiro lugar para homens, e em segundo para mulheres, s6 abaixo da Bulgária e da Hungria (sexo masculino) e da Bulgária (sexo feminino) ${ }^{25}$.

Nos Estados Unidos, a mortalidade por AVC vem caindo desde o início do século ${ }^{32,35}$, mas é possível que tenha havido um diagnóstico excessivo da doença como causa de morte no início do século. Uma grande proporçāo de mortes súbitas que, hoje, com os desenvolvimentos dos procedimentos diagnósticos para doenças cardiovasculares, é atribuída à doença coronária, pode ter sido anteriormente diagnosticada como "apoplexia"36.

Por outro lado, patologias encefálicas (tumor cerebral, hematoma subdural ou hidrocefalia de pressão normal) podem também ter sido erroneamente diagnosticadas como AVC e esta causa ter sido apresentada nos atestados de óbito como a causa básica da morte ${ }^{30}$. Nos países em desenvolvimento, este fenômeno de redução do "sobrediagnóstico" (overdiagnosis) pode estar ocorrendo agora e, eventualmente, os AVCs poderiam ser ainda uma "forma de morrer", isto é, uma afecção terminal mencionada como causa básica da morte em locais com precária assistência médica. Ao menos nos países desenvolvidos os hábitos diagnósticos parecem não ter sofrido alteração substancial desde os anos 40, e a mortalidade por AVC continua a cair apesar das alterações que pudessem advir das várias revisōes da Classificação Internacional de

* Departamento de Medicina Preventiva da Faculdade de Medicina da Universidade de São Paulo - Av. Dr. Arnaldo, 455 - 01246 - Sảo Paulo, SP - Brasil.

* Divisão de Clínica Médica do Hospital Universitário da Universidade de São Paulo - Cidade Universitária "Armando de Sales Oliveira" - 05508 - São Paulo, SP — Brasil.

** Departamento de Epidemiologia da Faculdade de Saúde Pública da Universidade de São Paulo - Av. Dr. Arnaldo, 715 - 01255 - São Paulo, SP - Brasil. 
Doenças. A pesar de todas estas considerações podese assumir que, desde 1940, a mortalidade vem declinando em alguns países, e este declínio é real, não devido a artefatos estatísticos ou meramente a formas diferentes de atestar a causa básica da morte.

O declínio nas taxas de mortalidade por AVC pode ser uma conseqüência do declínio na letalidade da doença, por melhor tratamento da mesma ou de suas complicaçōes, ou ainda, por casos menos severos, ou ser devido à queda da incidência dos novos casos de AVC, ou a uma combinação dos dois fatores.

A maior sobrevida após o AVC pode ser devida à melhora no tratamento ou ao fato de que os AVCs sejam de menor severidade clínica. Com relação ao primeiro fator, pode-se pensar que tenha havido um melhor cuidado de pacientes com complicações como insuficiência cardíaca e infecções respiratórias. É bem sabida a influência de infecçỗes respiratórias na sobrevida de pacientes com AVC. Com relação ao tratamento específico, não têm havido avanços substânciais para a maioria dos tipos de $\mathrm{AVC}^{23}$. Uma outra possibilidade para a maior sobrevida é a alteração da história natural do AVC. Alguns estudos têm mostrado uma menor proporção de pacientes comatosos ${ }^{3}$; outros têm relatado uma redução na incidência de hemorragias cerebrais, a forma mais letal de $\mathrm{AVC}^{23}$, mas nem sempre tal classificação é exata. Assim, mesmo considerando a ocorrência de avanços com relação ao tratamento das complicações, por si só tais melhoras não explicariam a queda de mortalidade. Por isso, têm-se sugerido que a diminuição na incidência e modificações na história natural da doença sejam os fatores determinantes para a redução da mortalidade ${ }^{14}$.

A pesar de diversas evidências com relação à queda da incidência, alguns autores discutem se este declínio é real e tentam estabelecer critérios de comparação entre os diferentes estudos para se obter tendências geográficas e seculares, e o único estudo conclusivo foi segundo eles, realizado em Rochester, onde se observou uma redução de $54 \%$ de incidência entre o primeiro e o último qüinqüênios de observação ${ }^{27}$. Nesse citado estudo, a incidência média anual de novos casos de AVC declinou em cada qüinqüênio desde 1950; comparando 1950-4 com 1970-4, o decréscimo na incidência foi de cerca de $50 \%{ }^{13}$. Entretanto, apesar da queda na incidência, mostrou-se que para o tipo mais comum de $A V C$, o infarto cerebral, a sobrevida de 7 anos após o episódio inicial mudou em 1945-9, em Rochester, de $22 \%$ para $40 \%$ em $1970-44$. Em Estocol$\mathrm{mo}^{4}$, mostrou-se pequeno aumento da incidência da AVC em homens durante o período 1974-81, enquanto a mortalidade decrescia no mesmo período à proporção de 2,3\% ao ano; nas mulheres, embora $a$ incidência não mostrasse alterações, a mortalidade caiu à proporção de 3,5\% ao ano. Em Wash- ington County, Estados Unidos, a incidência anual média ajustada por idade praticamente não mudou entre 1969-71 e 1974-6, mas houve um pequeno declínio na taxa de letalidade ${ }^{23}$.

$O$ fator de risco mais fortemente associado com os AVCs é a hipertensão arterial sistêmica( HAS). No estudo de Framingham ${ }^{19}$, o risco relativo de desenvolver trombose cerebral era de 4,6 vezes para os hipertensos em relação à população normotensa. Não havia nesse estudo um nível crítico de pressão arterial a partir do qual se pudesse considerar de maior risco, sendo o mesmo proporcional ao nível de pressão arterial sistólica e/ou diastólica. Os portadores de hipertensão arterial sistólica isolada tinham também riscos mais elevados do que os normotensos ${ }^{20}$. Tanto em Framingham quanto em Evans County (Estados Unidos) o grau de hipertensão foi um indicador muito mais seguro para a ocorrência de AVC do que de doença coronária ${ }^{16,20}$. Em outro estudo prospectivo, analisando uma coorte de homens nascidos em 1913, em Gotemburgo (Suécia), a pressão arterial elevada era o principal fator de risco para a ocorrência de $\mathrm{AVC}^{34}$.

O papel da HAS é confirmado por uma grande quantidade de ensaios clínicos nos quais a redução da incidência tem sido mostrada após terapêutica anti-hipertensiva. $O$ estudo "Medical Research Council" 28 trouxe evidências claras de que o tratamento da HAS leve reduz a incidência de AVC. $O$ "Hypertension Detection and Follow-Up Program"17, comparando um grupo de pacientes com tratamento clássico com outro submetido a tratamento mais intensivo, observou uma redução significativamente maior da incidência da doença no grupo com tratamento intensivo (incidência observada de 1,9 por 100 indivíduos) do que no grupo em tratamento convencional (2,9 por 100 indivíduos). O grupo sob observação mais rigorosa teve uma diminuição da mortalidade comparável à da população geral dos Estados Unidos.

Não se pode negar que a terapêutica para a HAS reduz tanto a mortalidade quanto a morbidade por AVC. Entretanto, pode-se discutir se este é o principal fator para explicar o declínio da mortalidade. Só nos anos 50 é que se tornaram disponíveis drogas anti-hipertensivas efetivas, e por isto mesmo, o controle da HAS não poderia explicar por si só o declínio que já vinha ocorrendo antes desta data. A taxa de declínio da mortalidade por AVC nos Estados Unidos, de 1920 a 1970 mantevese relativamente estável, em torno de $1,0 \%{ }^{30}$ a $2,0 \%{ }^{22}$. A pós 1970, ocorreu maior aceleração da queda, atingindo, após $1973,7,0 \%$ do ano 22 . Em outros países, mostrou-se da mesma maneira, uma aceleração para o declínio da mortalidade nos $\operatorname{anos} 70^{33}$.

Pode-se acompanhar paralelamente a evolução temporal da incidência no estudo, em Rochester ${ }^{13}$. A redução na incidência parece ter ocorrido em 
duas fases. De 1945-9 a 1955-9, o declínio médio anual global na incidência ajustada por idade e sexo foi de 3,1 por 100.000 habitantes. Houve um "platô" até 1964, quando então o declínio anual foi de 4,8 e 5,3 por 100.000 habitantes para os qüinqüènios 1965 9 e 1970-4, respectivamente ${ }^{13}$. Poder-se-ia, então, sugerir que o controle de hipertensão aumentou a taxa de declínio da mortalidade através de um declínio da incidência de AVC.

Embora uma proporção significativa de hipertensos permaneça sem terapêutica efetiva, a detecção, o tratamento e o controle da HAS vêm aumentando em todo o mundo $o^{5,12,26,35}$. A aceleração da queda na mortalidade por AVC tem sido associada com um aumento da proporção de hipertensos detectados, tratados e controlados na população. Alguns autores têm tentado quantificar quanto deste declínio poderia ser associado à terapêutica anti-hipertensiva. Um dos estudos apontou que o tratamento da HAS nos Estados Unidos ${ }^{6}$, no período 1970-4, poderia ser responsável por um declínio de $10 \%$ nas taxas de mortalidade (que é o mesmo declínio ajustado por idade da população americana no período). Entretanto, o mesmo estudo aponta que os pressupostos utilizados para estes cálculos eram criticáveis. Na Nova Zelândia, para o período 1973-82, estimou-se que o tratamento da HAS poderia ser responsável somente por cerca de $10 \%$ do total da redução de mortes por $\mathrm{AVC}^{5}$. Por outro lado, em outro estudo mais recente nos Estados Unidos ${ }^{22}$, não se achou correlação nítida do declínio da mortalidade por AVC com os dados obtidos nos três grandes estudos de prevalência de hipertensão arterial realizados naquele país .

Acaso outros fatores poderiam ser responsabilizados pelo declínio da mortalidade por AVC? Além do melhor controle da HAS, nos anos recentes, há estudos mostrando reduções em outros fatores de risco para doenças cardiovasculares nas populações, tais como nos níveis de colesterol sangüíneo e da prevalência do hábito de fumar ${ }^{26}$.

A relação do colesterol com a ocorrência de AVC não é consistente na literatura ${ }^{16,19,20,21,34}$. Contudo, recentemente o "Multiple Risk Factor Intervention Trial" (MRFIT) ${ }^{18}$ mostrou associação direta entre níveis elevados de colesterol e a mortalidade por AVCs isquêmicos e uma relação inversa entre estes níveis altos e a ocorrência de AVCs hemorrágicos. Muitos estudos anteriores não levam em consideração a classificação do AVC, e isto poderia explicar parte das inconsistências.

Em relação ao hábito de fumar, são controversas as associações entre este e o declínio da mortalidade por AVC, apesar de ser o hábito de fumar o único fator de risco para doenças cardiovasculares tão prevalente quanto a HAS. A coorte de Gotemburgo não achou associações consistentes para fumo e $\mathrm{AVC}^{34}$. Já o estudo do "Honnolulu Heart Pro- gram" 1 mostrou associações em homens e o "Nurse's Health Study" ${ }^{9}$ mostrou-as em mulheres. No estudo do "Medical Research Council" ${ }^{28}$ concluiu-se que tanto para os AVCs quanto para todos os eventos cardiovasculares, a diferença entre as taxas de fumantes e de não-fumantes era maior do que o efeito do tratamento medicamentoso.

Outros fatores de risco tais como a obesidade, principalmente a abdominal (do homem adulto), a ingestão de potássio na dieta, o uso de bebidas alcoólicas e o diabetes mellitus, dentre outros, têm sido citados para a ocorrência de AVC. Com relação ao diabetes mellitus, as associações são positivas $^{30}$. Com referência à obesidade, o estudo em Gotemburgo mostrou uma associação direta entre a incidência de AVC e a ocorrência de obesidade abdominal ${ }^{34}$.

A ingestão diâria de potássio tem sido investigada nos anos mais recentes, tendo-se mostrado que o consumo de alimentos ricos em potássio, tais como frutas e verduras, tem um efeito protetor com relação à mortalidade por $\mathrm{AVC}^{2}, 21$. Estas evidências, porém, não são ainda conclusivas. Tem-se descrito que o alto consumo de sódio em geral se acompanha de um baixo consumo de potássio ${ }^{31}$, mas é muito difícil avaliar se houve modificações na ingestão de sódio nos diferentes países no decorrer do tempo.

Em relação ao uso de álcool, sabe-se que ingestões moderadas ou baixas de bebida alcólica se associam com menor mortalidade por doenças cardiovasculares $^{10}$, mas discute-se se existem vícios de aferição da ingestão. Em dependentes de álcool, contudo, a prevalência de hipertensão arterial é também mais alta ${ }^{11}$, o que dificulta a análise, embora haja evidência de que a associação seja positiva ${ }^{15}$.

Outro ponto a ser considerado é a contribuição dada pela assistência à saúde para o declínio da mortalidade por AVC. Alguns autores têm sugerido que a maioria da mortalidade por AVC (assim como a mortalidade por doença coronária) vem ocorrendo fora do sistema de assistência médica, sendo que o declínio se relaciona a fatores ambientais, sociais ou culturais e a mudanças no comportamento relacionado à saúde ${ }^{6,8}$. Outros, contudo, acreditam que a aceleração da queda a partir dos anos 70 se deva à maior efetividade das ações de saúde ${ }^{30}$.

A despeito do progresso na identificação dos fatores de risco e dos esforços de identificação das causas do declínio na mortalidade por AVC, não existem ainda explicações suficientes para este. $O$ projeto patrocinado pela Organização Mundial da Saúde e que visa o monitoramento das tendências e dos determinantes de doenças cardiovasculares, em diversos países (Projeto MONICA) ${ }^{33}$, pode aumentar a compreensão das razões deste declínio. 
ELUF NETO, J. et al. [The treatment of hypertension and the decline of mortality from cerebrovascular accidents] Rev. Saúde públ., S. Paulo, 24: 332-6, 1990.

ABSTRACT: A possible cause-effect relation ship between the decline of mortality from cerebrovascular accidents (CVA) and a better control of arterial hypertension is discussed. The international literature on the subject is critically reviewed in the light of the possible statistical artifacts for enumeration of CVAs, the incidence and fatality of the disease, the prevalence of other risk factors such as hypercholesterolemia, smoking, and the consumption of sodium, potassium and alcohol, and obesity, as well as the contribution of health care.

KEYWORDS: Cerebrovascular desorders, mortality. Forecasting. Hypertension, prevention.

\section{REFERÊNCIAS BIBLIOGRÁFICAS}

1. ABBOTT, R. D. et al. Risk of stroke in male cigarette smokers. New Engl. J. Med., 315: 717-20, 1986.

2. ACHESON, R. M. \& WILlIAMS, D. R. R. Does consumption of fruit and vegetables protect against stroke? Lancet, 1: 1191-3, 1983.

3. AHMED, O. I. et al. Declining mortality from stroke in Allegheny County, Pennsylvania. Stroke, 19: 1814, 1988.

4. ALFREDSSON, L. et al. Mortality from and incidence of stroke in Stockholm. Brit. med. J., 292: 1299 1303,1986

5. BONTTA, R. \& BEAGLEHOLE, R. Does treatment of hypertension explain the decline in mortality from stroke? Brit. med. J., 292: 191-2, 1986.

6. BONITA, R. \& BEAGLEHOLE, R. The decline in stroke mortality; the limited role of antihypertensive therapy. N. Z. med. J., 100: 454-6, 1987.

7. BORHANI, N. O. Changes in geographic distribution of mortality from cerebrovascular disease. Amer. J. públ. Hlth, 55: 673-81, 1965.

8. BUCK, C. et al. Servicios de salud y politica de salud; discusion. In: Buck, C. et al., comp. El desafio de la epidemiologia: problemas y lecturas seleccionadas. Washington, D.C., Organización Panamericana de la Salud, 1988. p. 881-99. (OPAS-Publicación Cientifica, 505).

9 - COLDITZ, G. A. et al. Cigarette smoking and risk of stroke in middleaged women. New Engl. J. Med., 318: $937-41,1988$.

10. CRIQUI, M. H. Alcohol and cardiovascular mortality. In: Kaplan, R. M. \& Criqui, M. H., eds. Behavioral epidemiology and disease prevention. New York, Plenum Fress, 1985. p. 67-89.

11. FRASER, G. E. Preventive cardiology. New York, Oxford University Press, 1986. p. 142-3.

12. GARRAWAY, W. M. \& WHISNANT, J. P. The changing pattem of hypertension and the declining inci- dence of stroke. J. Amer. med. Ass., 258: 214-7, 1987.

13. GARRAWAY, W. M. et al. The declining incidence of stroke. New Engl. J. Med., 300: 449-52, 1979.

14. GARRAWAY, W. M. et al. The changing pattern of survival following stroke. Stroke, 14: 699-702, 1986.

15. GILL, J. S. et al. Stroke and alcohol consumption. New Engl. J. Med., 315: 1041-6, 1986.

16 HEYMAN, A. et. al. Cerebrovascular disease in the biracial population of Evans County, Georgia. Arch. intern. Med., 128: 949-55, 1971.

17. HYPERTENSION DETECTION AND FOLLOW-UP PROGRAM COOPERATIVE GROUP. Five-year findings of the Hypertension Detection and Follow-up Program. J. Amer. med. Ass., 247: 633-8, 1982.

18. ISO, H. et al. Serum cholesterol levels and six-year mortality from stroke in 350,977 men screened for the multiple risk-factor intervention trial. New Engl. J. Med., 320: 904-10, 1989.

19. KANNEL, W. B. et al. Epidemiologic assessment of the role of blood pressure in stroke - The Framingham Study. J. Amer. med. Ass.,2 14: 301-10, 1970.

20. KANNEL, W. B. et al Systolic blood pressure, arterial rigidity and risk of stroke - The Framingham Study, $J$. Amer. med. Ass., 245:1225-9, 1981.

21. KHAW, K. T. \& BARRETT-CONNOR, E. Dietary potassium and stroke - associated mortality. A 12-year prospective population study. New. Engl. J. Med., 316: 235-40, 1987.

22. KLAG, M. J. et al. Decline in US stroke mortality: demographic trends and antihypertensive treatment. Stroke, 20: 14-21, 1989.

23. KRAMER, $S$. et al. Patterns of incidence and trends in diagnostic classification of cerebrovascular disease in Washington County, Maryland, 1969-1971 to 1974-76. Amer. J. Epidem., 115: 398.411, 1982. 
24. LOLIO, C. A. de \& LAURENTI, R. Tendências na mortalidade por doenças cerebrovasculares em adultos acima da idade de 20 anos em São Paulo, Estado de São Paulo, Brasil, 1950 a 1981. Rev. Saúde públ., S. Paulo, 20: 343-6, 1986.

25. LOLIO, C. A. de et al. Decline in cardiovascular disease mortality in the city of S. Paulo, Brasil, 1970 to 1983. Rev. Saúde públ., S. Paulo, 20: 454-64, 1986.

26. LUEPKER, R. V. et al. Cardiovascular risk factor change - 1973-1974 to 1980-82: the Minnesota Heart Survey. J. clin. Epidem., 41: 825-33, 1988.

27. MALMGREN. R. et al. Geographical and secular trends in stroke incidence. Lancet, 2: 1196-1200, 1987.

28. MEDICAL RESEARCH COUNCIL WORKING PARTY. MRC trial of treatment of mild hypertension: principal results. Brit. med. J., 291:97-104, 1985.

29. MINISTÉRIO DA SAÚDE. Estatísticas de morlalidade: Brasil, 1985. Brasília, Centro de Documentação do Ministério da Saúde, 1988.

30. OSTFELD, A. A review of stroke epidemiology. Epidem. Rev., 2: 136-52, 1980.
31. PAGE, L. B. Epidemiology of hypertension. In: Genest, J., ed. Hypertension. 2nd. ed. New York, McGraw Hill, 1983. p. 683-99.

32. SOLTERO, I. et al. Trends in mortality from cerebrovascular disease in the United States, 1960 to 1975. Stroke, 9: 549-55, 1978.

33. UEMURA, K. \& PISA, Z. Recent trends in cardiovascular disease mortality in 27 industrialized countries. Wld Hlth Statist. Quart., 38: 142-57, 1985.

34. WELIN, L. et al. Analysis of risk factors for stroke in a cohort of men born in 1913. New Engl. J. Med., 317: $521-6,1987$.

35. WHISNANT, J. P. The decline of stroke. Stroke, 15: $160-8,1984$.

36. WYLIE, C. M. Recent trends in mortality from cerebrovascular accidents in the United States. $J$. chron. Dis., 14: 213-20, 1961.

Recebido para publicaçäo em 29/11/1989 Aprovado para publicação em 17/4/1990 